\title{
A New Nutation Model Of Nonrigid Earth With Ocean And Atmosphere
}

\author{
Cheng-li Huang, Wen-jing Jin, Xing-hao Liao \\ Shanghai Astronomical Observatory, UCAR, NAO, CAS, Shanghai, \\ 200030, P.R.China (email: clhuang@center.shao.ac.cn)
}

\begin{abstract}
By integrating the truncated complex scalar gravitational motion equations for an anelastic, rotating, slightly elliptical Earth, the complex frequency dependent Earth transfer functions are computed directly. Unlike the conventional method, the effects of both oceanic loads and tidal currents are included via outer surface boundary conditions, all of which are expanded to second order in ellipticity. A modified ellipticity profile in second order accuracy for the non-hydrostatic Earth is obtained from Clairaut's equation and the PREM Earth model by adjusting both the ellipticity of the core-mantle boundary and the global dynamical ellipticity to modern observations. The effects of different Earth models, anelastic models, and ocean models are computed and compared. The atmospheric contributions to prograde annual, retrograde annual and retrograde semiannual nutation are also included as oceanic effects. Finally, a complete new nutation series of more than 340 periods, including inphase and out-of-phase parts of longitude and obliquity terms, for a more realistic Earth, is obtained and compared with other available nutation series and observations.
\end{abstract}

\section{Introduction}

Usually, the methodology of the nonrigid-Earth nutation study fall into four categories: (1) completely fitted from modern observation (VLBI plus LLR) (McCarthy, 1996); (2) semi-analytical method (Mathews et al., 1991, 2000); (3) based on the Hamiltonian theory and variational principles, extending the method of rigid Earth nutation study to the study of nonrigid Earth nutation (Getino \& Ferrándiz, 1999); and (4) purely geophysical approaches, in which the so-called nonrigid Earth nutation transfer function is integrated from the equations of Earth motion by a normal mode expansion method (Wahr 1981, Dehant \& Defraigne 1997) or by a direct numerical integration method (Schastok 1997, Huang 1999), which is used in this study again.

\section{The numerical integration method}

Smith (1974) developed an algorithm, in which the scalar, but coupled due to the ellipticity, ordinary equations, as well as a set of boundary conditions, governing the elastic-gravitational normal modes of a rotating, slightly elliptical Earth in 
hydro-static equilibrium, having an isotropic elastic constitutive relation, are finally derived, truncated to finite order and described in functional forms, on which are based almost all the modern nutation and tides theories for a nonrigid Earth.

The given equations of motion are in ordinary differential form. In order to overcome the resonance "pollution" and to detect small signals, such as the contributions of atmosphere and oceanic current, Schastok (1997) and Huang (1999) re-derived the boundary conditions about the stress tensor and gravitational potential field to second order, instead of first order given by Smith (1974), in ellipticity, as well as the boundary conditions about the displacement field (Huang, 1999; Huang, 2000a).

\subsection{The contributions of the ocean and atmosphere}

Unlike the conventional method, the effects of atmospheric relative motions, as well as the oceanic load and current effects, are included via non-free outer surface boundary condition in this paper.

If the Earth does not include ocean and atmosphere, the outer surface of the solid Earth is a free one, i.e. the pressure and the stress tensor at the outer boundary of the solid Earth cancel in the integration. On the contrary, if the ocean and/or atmosphere are included in the Earth system, the outer surface of the solid Earth is no longer a free one. The stress tensor at the outer boundary of the solid Earth is given by Sasao \& Wahr (1981) in tensor format and expanded into scalar format of three components $\left(P_{l}^{m}, Q_{l}^{m}, R_{l}^{m}\right)$ to second order in ellipticity by Schastok (1997) and Huang (1999). In their expressions of the continuity quantity at the non-free outer surface boundary, there are two quantities related to the atmospheric (or oceanic) contribution, $h_{l}^{m}(a, \omega)$ and $\Lambda_{l}^{m}(a, \omega)$, which represent the relative angular momentum and the load respectively, where $a$ and $\omega$ denote the mean radius of the Earth and the circular frequency of the nutation, and $l$ and $m$ are the degree and order of GSSH.

We adopt the model of Zahel (1995), which gives both the amplitudes and the phases of several dominant diurnal tides of the load and the relative motion separately, and an empirical function, linear in frequency, to interpolate and extrapolate the other diurnal tides as Schastok (1997) did. However, the atmospheric spectrogram is usually contaminated strongly and there is not any atmospheric model which can give the amplitude and the phase of the corresponding diurnal atmospheric tides.

In this paper, we adopt the concept of celestial effective angular momentum (CEAM) to discuss in the frequency domain. The EAM in TRF, $\chi$, can be converted pointwise to the $\mathrm{CRF}, \chi^{\prime}$, by multiplying by a factor of the sidereal rotation, $e^{i \Phi}$ (or $e^{i \Omega t}$ ) (Brzeziński, 1994).

The original near-diurnal signals in the terrestrial reference frame (TRF) become low-frequency signals in the celestial reference frame (CRF); on the contrary, the original long-period signals in the TRF become high-frequency signals in the space. Then we can use a low-pass filter to keep the low-frequency signals of $\chi^{\prime}$ and filter out the high-frequency part easily (Bizouard et al., 1998). Huang et al. (2000b) processed the atmospheric EAM data from 1980.0 to 2000.0 provided by NCEP in which there are four evenly distributed points 


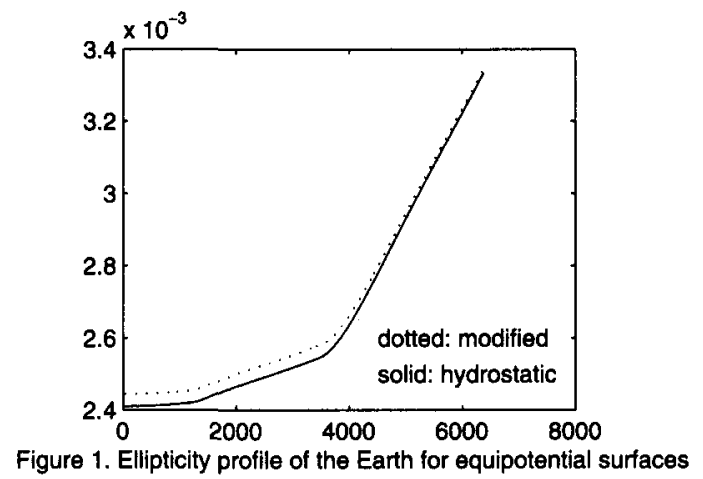

Figure 1. Ellipticity profile of the Earth for equipotential surfaces.

everyday. That study assumes that the oceanic reaction to the atmosphere is non-inverted-barometer (NIB) because it is more true for the high frequency variation likes diurnal tides. The amplitudes of the long-trend term and three periods from standard least-squares fit as well as the corresponding relative angular momentum, $h$, caused by the wind are given in that paper and are used in this study.

\subsection{Inelastic mantle}

If the mantle inelasticity is considered, the Lamé coefficients become frequency dependent and complex, and will produce out-of-phase contributions to nutation (Dehant, 1987). A frequency-to-the-power- $\alpha$ model (Anderson \& Minster, 1979) is used to compute them in this study. The radially dependent quality factor, Q, is taken from the anelasticity model QMU of Sailor \& Dziewonski (1978), and the reference frequency $\omega_{0}$ and the power $\alpha$ are taken as 200s and 0.15 respectively (Wahr \& Bergen, 1986).

\subsection{The ellipticity profiles of the Earth interior}

In addition to the density profile, the ellipticity profiles of the Earth's interior are very important for the nutation computation, although they are less well-known.

The ellipticity profiles of the Earth's interior depend on the density profile and can be obtained from the second order Clairaut's equation for the Earth in hydrostatic equilibrium, which is plotted as a solid line in Figure 1.

In order to be consistent with modern observations, we assume that the CMB and two discontinuities $(\mathrm{r}=5701$ and $5971 \mathrm{~km}$ ) are not in hydrostatic equilibrium using two constraints: (1) the resultant $H$ is consistent with the observed value, i.e. $H^{\text {obs }} \approx 0.00327379$ while from PREM (Dziewonski \& Anderson, 1981$), H^{P R E M} \approx 0.003240$, and $(2)$ the resultant retrograde FCN period (real part) is -432.8 sidereal days (with ocean), while it is about -458 days for PREM. In order to satisfy this requirement, $\epsilon_{C M B}$ is modified from 0.002547 
(for PREM) to 0.002666 , while the $\epsilon_{5701}$ is modified to 0.003200 and $\epsilon_{5971}$ is modified to 0.003224 . The modified ellipticity profile of the equipotential surfaces can then be obtained from Clairaut's equation and plotted in Figure 1 also (dotted line).

As a result, the period of the prograde free inner core nutation (FICN) is also obtained, which is +465.6 sidereal days (with ocean) for modified ellipticity profile and is +470.6 sidereal days for PREM model.

\section{Results and comparison}

By integrating the truncated complex scalar gravitational motion equations, the complex Earth transfer functions for each nutation frequency are computed directly and are then convolved with the rigid-Earth nutation series REN2000 (Souchay et al., 1999). In the integration, the density profile of the Earth model PREM is used, and the normal step length of the integration is $5 \mathrm{~km}$. Finally, the resulting nonrigid-Earth nutation series of 343 terms, in which the effects of the ocean load and current and atmospheric winds are included, are calculated and available for interested readers by anonymous FTP server (ftp://center.shao.ac.cn/clhuang/nutation/hj12000-343.txt) or by contacting the authors, because it is too long to present in this paper.

Moreover, the main four nutation terms (18.66 years, 1 year, 1/2 year and 13.66 days) have been compared with other available observational and theoretical series (see Table 1). They are: (1) IERS Conventions (1996) (McCarthy, 1996) nutation series (abbreviated as IERS96 hereafter) fitted from VLBI observations by Dr. T. Herring ; (2) Dehant \& Defraigne (1997) model (DD97); (3) Schastok (1997) model (S97); (4) Getino \& Ferrándiz (1999) model (GF99); and (5) Mathews et al. (2000) model (MBH2000). 
Table 1. Comparison of the four main nutation terms (units: mas)

\begin{tabular}{|c|c|c|c|c|}
\hline & $\delta \psi_{i p}$ & $\delta \psi_{o p}$ & $\delta \epsilon_{o p}$ & $\delta \epsilon_{i p}$ \\
\hline \multicolumn{5}{|c|}{$18.66 \mathrm{yr}$} \\
\hline IERS96 & -17206.277 & 3.645 & 1.553 & 9205.356 \\
\hline $\mathrm{S} 97$ & -17206.35 & 2.28 & 1.11 & 9205.05 \\
\hline DD97 & -17205.39 & - & - & 9204.87 \\
\hline GF99 & -17206.251 & 3.235 & 1.450 & 9205.403 \\
\hline MBH2000 & -17206.458 & 3.334 & 1.533 & 9205.175 \\
\hline This paper & -17206.271 & 3.267 & 1.309 & 9205.147 \\
\hline \multicolumn{5}{|c|}{$1 \mathrm{yr}$} \\
\hline IERS96 & 147.538 & 1.121 & -0.198 & 7.388 \\
\hline $\mathrm{S} 97$ & 147.66 . & -0.24 & +0.14 & 7.27 \\
\hline DD97 & 147.52 & - & - & 7.40 \\
\hline GF99 & 147.550 & 1.247 & -0.445 & 7.256 \\
\hline MBH2000 & 147.557 & 1.197 & -0.203 & 7.400 \\
\hline This paper & 147.567 & 0.250 & -0.173 & 7.423 \\
\hline \multicolumn{5}{|c|}{0.5 уг } \\
\hline IERS96 & -1317.014 & -1.400 & -0.464 & 573.058 \\
\hline S97 & -1316.95 & -1.42 & -0.43 & 572.95 \\
\hline DD97 & -1316.94 & - & - & 572.95 \\
\hline GF99 & -1317.028 & -1.749 & -0.625 & 573.005 \\
\hline MBH2000 & -1317.159 & -1.382 & -0.459 & 573.061 \\
\hline \multicolumn{5}{|c|}{$13.66 \mathrm{~d}$} \\
\hline IERS96 & -227.720 & 0.269 & 0.136 & 97.864 \\
\hline S97 & -227.71 & 0.37 & 0.18 & 97.86 \\
\hline DD97 & -227.82 & - & 一 & 97.90 \\
\hline GF99 & -227.733 & -0.407 & -0.153 & 97.869 \\
\hline $\mathrm{MBH} 2000$ & -227.588 & 0.333 & 0.163 & 97.814 \\
\hline This paper & -227.718 & 0.328 & 0.178 & 97.858 \\
\hline
\end{tabular}

Acknowledgments. We are indebted to Drs. J. Schastok and V. Dehant for their judicious discussions and kindly assistance.

\section{References}

Anderson, D.L., Minster, J.B., 1979, Geophys. J. R. Astron. Soc., 58, 431.

Bizouard, C., Brzeziński, A., Petrov, S., 1998, J. Geodesy, 72, 561.

Brzeziński, A., 1994, Manuscripta Geodetica, 19, 157.

Dehant, V., 1987, Phys. Earth Planet. Inter., 49, 242.

Dehant, V., Defraigne, P., 1997, J. Geophys. Res., 102, 27659.

Dziewonski, A.D., Anderson, D.L., 1981, Phys. Earth planet. Inter., 25, 297.

Getino, J., Ferrándiz, J.M., 1999, M.N.R.A.S., 306, L45. 
Huang, C.-L., 1999, A study of the nutation of non-rigid Earth with ocean and atmosphere, Ph.D. thesis, 148 pp., Shanghai Astronomical Observatory, Chinese Academy of Sciences, August, 1999.

Huang, C.-L., 2000a, A scalar boundary condition for the motion of the elastic Earth to second order in ellipticity, submitted to Earth Moon and Planets.

Huang, C.-L., Liao, X.-H., Jin, W.-J., 2000b, The contribution of the wind to the Earth nutation by numerical integration method, submitted to Geophys. Res. Lett.

Mathews, P.M., Buffett, B.A., Herring, T.A., 1991, J. Geophys. Res., 96, 8219. Mathews, P.M., Buffett, B.A., Herring, T.A., 2000, this issure.

McCarthy, D.D., ed., 1996, IERS Conventions (1996).

Sailor, R.V., Dziewonski, A.M., 1978, Geophys. J. R. Astron. Soc., 53, 559.

Sasao, T., Wahr, J.M., 1981, Geophys. J. R. Astron. Soc., 64, 729.

Schastok, J., 1997, Geophys. J. Int., 130, 137.

Smith, M. L., 1974, Geophys. J. R. Astron. Soc., 37, 491.

Souchay, J., Loysel, B., Kinoshita, H., et al., 1999, Astron, Astrophys. Suppl., $135,111$.

Wahr, J. M., 1981, Geophys. J. R. Astron. Soc., 64, 651.

Wahr, J.M., Bergen, Z., 1986, Geophys. J. R. Astron. Soc., 87, 633.

Zahel W., 1995, J. Mar. Systems., 6, 3. 